\title{
Analysis of Misregistration Induced Color Shifts in the Superposition of Periodic Screens
}

\author{
Basak Oztan $^{a}$, Gaurav Sharma ${ }^{a}$ and Robert P. Loce $^{b}$ \\ ${ }^{a}$ University of Rochester, Rochester, NY, USA \\ ${ }^{b}$ Xerox Corporation, Webster, NY, USA
}

\begin{abstract}
We present an analysis and model for evaluation of color shifts in halftone printing caused by inter-separation misregistration for periodic clustered dot halftones. Using a lattice framework, we present intuitive analysis that demonstrates conditions under which the average color is asymptotically invariant under inter-separation misregistration. Combining the framework with an analytic representation for the halftone dots, we develop a hybrid analytical-numerical model for quantitatively estimating color shifts as a function of inter-separation misregistration. The model is compared against experimental data for a xerographic printer.
\end{abstract}

Keywords: Misregistration in color printing, clustered dot halftoning, halftone lattice analysis, average color shift

\section{INTRODUCTION}

Color halftoning is commonly employed in hardcopy printing, wherein a continuous tone (contone) color image is reproduced with an overlay of bi-level (on/off) colorant images such that at normal viewing distances, the perception of the halftone image closely approximates that of the contone image. In typical color printing, Cyan (C), Magenta (M), Yellow (Y) and Black (K) colorants are used and each colorant channel is halftoned independently of the other channels. The printed image is then produced by sequentially printing the individual separation halftones in overlay on the paper substrate.

For ideal block-dye CMY colorants, ${ }^{1,2}$ one could design the halftones for the individual colorants independently because the colorants act on different regions of the spectrum and do not interact with one another. In actual practice, however, the colorants are always non-ideal and therefore interact. The interactions among colorants are best understood physically by considering each individual wavelength in the spatial Fourier domain*. To a first order of approximation, the subtractive overlay of separations combines their spectral transmittances multiplicatively. For any wavelength that is absorbed by more than one colorant, this multiplicative combination of the spatial halftone patterns produces Fourier spectrum components at sum and difference combinations of the frequency components in the individual halftones ${ }^{\dagger}$. In the choice of CMYK separation halftones, we must therefore avoid choices that are good for the individual separations but result in undesirable (i.e. visible) low frequency components in overlay.

Most color halftone methods and systems are based on the aforementioned general principle of avoiding halftone combinations that produce undesirable low frequency interactions. A component at zero spatial frequency (commonly referred to as the "d.c. component") has, however, often not been considered undesirable. One reason for this apparent anomaly is that the color control of the printer in fact eliminates the impact of a fixed d.c. component through the process used to map desired color values to printer CMYK combinations. However, the response at d.c. (and in fact any frequency) depends not only on the frequency components in the individual separation halftones, but also on their phase. Spatial misregistration between the color separations,

Send correspondence to B. Oztan: E-mail: basak@ece.rochester.edu, Telephone: 1 585 275-8122, Address: Electrical and Computer Engineering Department, University of Rochester, Rochester, NY, 14627-0126, USA, WWW: www.ece.rochester.edu/projects/iplab

This work was supported in part by a gift from the Xerox Foundation

${ }^{*}$ This is a spatial Fourier spectrum per wavelength in the (visible) electromagnetic spectrum.

†This follows directly from the so-called "modulation property" of the Fourier Transform.

Color Imaging XI: Processing, Hardcopy, and Applications, edited by Reiner Eschbach, Gabriel G. Marcu, Proc. of SPIE-IS\&T Electronic Imaging, SPIE Vol. 6058, 60580X, @ 2005 SPIE-IS\&T · 0277-786X/05/\$15 
corresponds to a change in relative phase and can therefore alter the value of the d.c. term if present, which is manifested as a color shift. Due to the mechanical paper transport involved in the printing process, some misregistration between the separations is unavoidable. In the present work, we focus on this misregistration induced color shift by examining the zero spatial frequency component produced in the overlay. Our goal is to examine the impact of misregistration both analytically and by means of a suitable simulation framework.

The preceding discussion is applicable to almost all methods that halftone color separations independently. Several techniques have been proposed for the halftoning of individual separations (or equivalently monochrome images) that fall under one of the following three categories ${ }^{3}$ : (1) point processes, (2) neighborhood algorithms and (3) iterative methods. The characteristics of the printing technology, specific application requirements, and computational complexity are some of the common factors that impact the choice of a halftoning technique. Due to their stability and predictability clustered-dot halftones, which belong to the class of point processes and mimic Talbot's original photographic screening process, ${ }^{4}$ are commonly used in the two primary methods in high volume printing: lithography and xerography. Conventional clustered dot halftones are amplitude modulated (AM) signals in the sense that different gray levels are reproduced by varying the dot sizes while keeping the periodicity of dots constant. In this work we restrict our attention to xerographic printers, thus consider only clustered-dot periodic halftones in our analysis of color variation with misregistration.

The remainder of this paper is organized as follows. We first present related work in Sec. 2 where we present a summary of the literature on this problem and make connections with our development. Then in Sec. 3 we develop our lattice framework and notation. In Sec. 3.2, we present asymptotic (infinite area) analysis of halftones to determine the conditions for misregistration sensitivity/insensitivity. Based on the development, we also propose an intuitive metric for estimation of misregistration insensitivity. Next, in Sec. 3.3 we incorporate an analytic model for the halftone threshold function in our lattice framework to develop a numerical model for quantitative estimation of color shifts due to misregistration in color halftones. We present results from the model along with comparisons against experimental data in Sec. 4. Finally, in Sec. 5 we present conclusions and a discussion.

\section{RELATED WORK}

The average spectrum of the halftone image may be modeled (to a first order) using the Neugebauer equations. ${ }^{5,6}$ Using these equations, we can readily see that if the areas of overlap between the separations can be modeled as statistically random, i.e., satisfy the Demichel equations, ${ }^{7}$ the average spectrum is independent of the inter-separation alignment. Randomization of the inter-separation overlaps therefore eliminates the problem of misregistration induced color shift in color halftones. With this motivation, rotated halftone screens in which the halftones for different separations are rotated relative to each other are commonly utilized in practice. Though, alternatives to the Demichel equations have been proposed for non-rotated halftone configurations, ${ }^{8}$ the validity of the Demichel equations for the common rotated screen configurations has not received much attention until relatively recently.

Rogers ${ }^{9}$ examined the validity of Demichel equations for the superposition of two orthogonal circular dot halftones having the same frequency and demonstrated that the equations hold only for certain angular separations between the screens. Rogers achieved this result by exploiting the circular symmetry of the dots and deriving an expression for the neighbor distribution of halftone dots.

Amidror et al. ${ }^{10}$ extend the work to the superposition of an arbitrary number screens. They present a general proposition that characterizes (in necessary and sufficient form) the failure of the Demichel equations in the Fourier domain by a singular configuration of frequency vectors ${ }^{\ddagger}$ Using computer simulations on a high resolution pixel grid, they numerically demonstrate the validity of the proposition. ${ }^{10}$ In particular, they establish that the conventional equi-frequency, $30^{\circ}$ angular separation, $\mathrm{C}, \mathrm{M}, \mathrm{K}$ halftone configuration used in lithographic printing $^{1}$ is not invariant to color misregistration. In related work, ${ }^{11}$ they also consider the stability of the rosette micro-structure resulting from the overlap of multiple screens and demonstrate that the micro-structure is periodic for singular configurations and aperiodic for non-singular configurations.

\footnotetext{
${ }^{\ddagger}$ We provide a precise mathematical definition of the term singular in the next section.
} 
In the first part of our work, we build on the foundation of Rogers ${ }^{9}$ and Amidror et al. ${ }^{10,11}$ We cast the general $K$-screen superposition problem in a lattice framework, that has previously been used for the frequency analysis of halftone superpositions. Each halftone separation is modeled as a periodic function with periodicity determined by a two-dimensional lattice. We show that under suitable constraints on the individual lattices (which always hold for a finite resolution device), the inter-separation dot-distances are also distributed on a two-dimensional lattice. Using the distribution, we obtain histograms for inter-separation dot distances analogous to Rogers. We then demonstrate that the Demichel equations apply under suitable constraints in the lattice framework that are in agreement with proposition of Amidror et al. We present the results using 1-dimensional illustrative examples that provide an intuitive insight in the lattice methodology. Based on the insight, we also propose a metric for the evaluation of misregistration sensitivity.

In the second part of our work, we develop a quantitative model for the estimation of color shift due to color misregistration. Prior work in this area has utilized computer simulations on a high resolution digital grid with pixel counting methods to estimate individual colorant and overlap areas. In addition to the work by Amidror et al $^{10}$ mentioned earlier, this approach has been used by Daels et al. ${ }^{12}$ to show the color shift between dot-centered and hole-centered rosettes for the conventional 3-screen rotated configuration and by Oztan et al. ${ }^{13}$ for quantitative estimates of color shifts due to misregistration for 2 colorant dot-on-dot, dot-off-dot and conventional rotated screens. The approach however has a limitation in accuracy due to the finite grid utilized and can also be very memory intensive for certain configurations. Instead, we develop a model that utilizes an analytic description of the halftone dot and allows direct numerical computation of the area of overlap between the dots. This allows the model to operate without the computationally expensive high resolution simulation allowing both faster computations and for more screen configurations.

\section{A FRAMEWORK FOR ANALYSIS OF COLOR HALFTONE MISREGISTRATION}

For the analysis of average color shift with misregistration, we use the system model illustrated in Fig. 1. We begin with a spatially invariant contone image consisting of $K$ colorant planes and model the color of the print resulting from a halftone obtained from this image. Though we adopt a generalized notation of $K$ colorants, throughout we consider only the common case of CMYK colorants, for which $K=4$. We assume the scenario mentioned in Section 1, wherein the $K$ colorant planes are independently halftoned using periodic clustered dot screens and printed in overlay. In general, the 2-D periodicity of the $i^{t h}$ separation may be represented using a planar lattice $\boldsymbol{\Lambda}_{i}$ in $\mathbb{R}^{2}$, mathematically defined as ${ }^{14}$

$$
\mathbf{\Lambda}_{i}=\left\{\mathbf{V}_{i} \mathbf{n} \mid \mathbf{n} \in \mathbb{Z}^{2}\right\}
$$

where $\mathbf{V}_{i}=\left[\mathbf{v}_{1}^{i} \mid \mathbf{v}_{2}^{i}\right]$ is the matrix of the linearly independent vectors $\mathbf{v}_{1}^{i}=\left[\begin{array}{c}v_{x_{1}}^{i} \\ v_{y_{1}}^{i}\end{array}\right], \mathbf{v}_{2}^{i}=\left[\begin{array}{c}v_{x_{2}}^{i} \\ v_{y_{2}}^{i}\end{array}\right]$ and $\mathbb{Z}$ denotes the set of integers. Thus $\boldsymbol{\Lambda}_{i}$ is the (discrete) set of all integer linear combinations of the vectors $\mathbf{v}_{1}^{i}$ and $\mathbf{v}_{2}^{i}$ in $\mathbb{R}^{2}$. The vectors $\mathbf{v}_{1}^{i}$ and $\mathbf{v}_{2}^{i}$ are said to form a basis for the lattice $\boldsymbol{\Lambda}_{i}$ and for any point $\mathbf{V}_{i} \mathbf{n}$ in the lattice, the vector $\mathbf{n}=\left[\begin{array}{l}n_{1} \\ n_{2}\end{array}\right]$ is the representation of the point in the lattice with respect to the basis $\mathbf{V}_{i}$.

The halftone for each colorant plane can now be modeled as the convolution of a halftone dot function with the corresponding periodic lattice in the spatial domain. ${ }^{15,16}$ To incorporate misregistration of separation $i$ the halftone dot function may be displaced by a displacement $\mathbf{d}=\left[\Delta x_{i}, \Delta y_{i}\right]$. In the print, these multiple halftone layers are overlaid producing all possible $2^{K}$ super-positions of the $K$ colorants, which are referred to as the Neugebauer primaries. Using the Neugebauer model, ${ }^{5,6}$ the average spectrum of the printed halftone is

$$
R_{\text {avg }}(\lambda)=\left(\sum_{c_{1}=0}^{1} \cdots \sum_{c_{1}=0}^{1} a_{c_{1} \ldots c_{K}} R_{c_{1} \ldots c_{K}}^{\frac{1}{\gamma}}(\lambda)\right)^{\gamma},
$$

where the $K$-bit binary index string $c_{1} \ldots c_{K}$ with $c_{j} \in\{0,1\}, \forall j=1,2, \ldots K$ represents one of the $2^{K}$ Neugebauer primaries in a convenient notation: $c_{l}$ indicates the presence of the $l^{\text {th }}$ colorant, i.e. the Neugebauer primary $c_{1} \ldots c_{l} \ldots c_{K}$ includes the $l^{t h}$ colorant in the overlap that constitutes it if and only if $c_{l}=1 . R_{c_{1} \ldots c_{K}}(\lambda)$ is then 


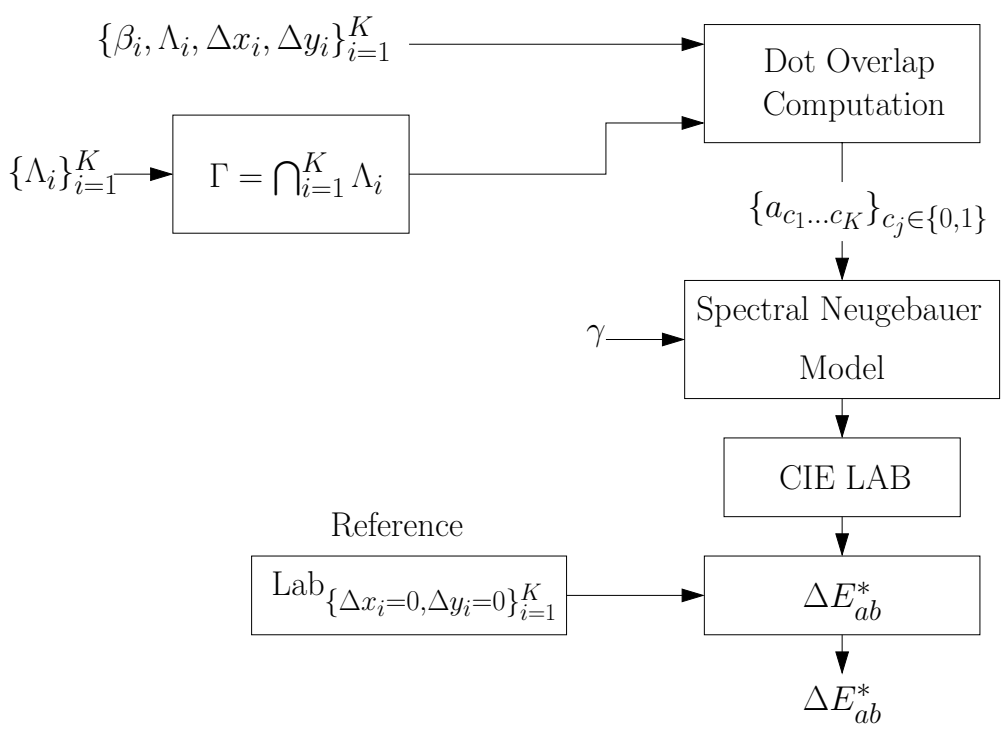

Figure 1. Overview of the analysis

the reflectance of the Neugebauer primary corresponding to the binary index $c_{1} \ldots c_{K}, a_{c_{1} \ldots c_{K}}$ represents the area covered by the corresponding primary as a fraction of the total area and $\gamma$ is the empirical Yule-Nielsen correction factor.

Now if we denote by $\beta_{j}$ the fraction of the total area covered by the $j^{\text {th }}$ colorant (which is possibly covered by additional colorants and therefore distinct from the area for the corresponding primary), we have

$$
\beta_{j}=\sum_{\left\{c_{1} \ldots c_{K} \mid c_{j}=1\right\}} a_{c_{1} \ldots c_{K}}
$$

We extend this notation and use $\beta_{j_{1} \ldots j_{m}}$ to represent the fractional area covered by colorants $j_{1} \ldots j_{m}$, where $j_{1} \ldots j_{m}$ is a set of distinct colorant indices in ascending order, i.e., $j_{1} \ldots j_{m} \in\{1, \ldots K\}$ and $j_{1}>j_{2}>\cdots>j_{m}$. Then, clearly

$$
\beta_{j_{1} \ldots j_{m}}=\sum_{\left\{c_{1} \ldots c_{K} \mid c_{j_{1}}=1, \ldots c_{j_{m}}=1\right\}} a_{c_{1} \ldots c_{K}}
$$

The above system of equations can be inverted to obtain expression for the fractional areas for the primaries $a_{c_{1} \ldots c_{K}}$ in terms of the fractional areas $\beta_{j_{1} \ldots j_{m}}$ for the colorant combinations. The same relation can also be obtained by the physical relation between these quantities based on their definitions. One can trivially see that $a_{1 \ldots 1}=\beta_{1 \ldots K}$ and

$$
a_{c_{1} \ldots c_{K}}=\beta_{\mathbf{j}(\mathbf{c})}-\sum_{\mathbf{j} \in \mathcal{I}^{+}(\mathbf{c})} \beta_{\mathbf{j}}
$$

where $\mathbf{c}$ denotes $c_{1} \ldots c_{K}$, the notation $\mathbf{j}(\mathbf{c})=j_{1} \ldots j_{m}$ denotes the string of indices $\left\{j_{l}\right\}_{l=1}^{m}$ for which $c_{j_{l}}$ is non-zero, arranged in ascending order, and $\mathcal{I}^{+}(\mathbf{c})$ is the set of all $m+1$ indices (in increasing order) that include the indices $j_{1} \ldots j_{m}$ in $\mathbf{j}(\mathbf{c})$ and one additional (distinct) index ${ }^{\S}$.

\footnotetext{
${ }^{\S}$ Note that each element of $\mathcal{I}^{+}(\mathbf{c})$ denotes a combination of $m+1$ colorant combinations that include the $m$ colorants indicated by $\mathbf{c}$ as a subset. Thus the summation in (5) represents the fractional area coverage of all distinct $m+1$ colorant combinations that include the $m$ colorants indicated by $\mathbf{c}$ as a subset.
} 
We saw above that the fractional areas $a_{c_{1} \ldots c_{K}}$ in the Neugebauer model of (2) can be computed in terms of the areas of the type $\beta_{\mathbf{j}}$, which corresponds to the fractional area that is common to (or covered by) the colorants indicated by the indices $\mathbf{j}$. The areas $\beta_{1}, \beta_{2}, \ldots \beta_{K}$ represent the fractional area covered by each of the individual colorants. These depend on the periodicity lattices for the colorants and the halftone dot functions, the latter also capture the dependence on the contone value for the colorant. The areas $\beta_{1}, \beta_{2}, \ldots \beta_{K}$ are independent of any amount of misregistration in any of the separations. However, the $\beta_{\mathbf{j}}$ terms corresponding to areas common to more than one colorant, in general, depend on the individual lattices, the corresponding spot functions, as well as the inter-separation mis-alignment. A simple illustrative example is the case of two separations with a spot function that corresponds to half a lattice cell and identical lattices for the two separations. In this case in the absence of misregistration $\beta_{12}=\beta_{1}=\beta_{2}$ however in the presence of a displacement corresponding to half the lattice period, $\beta_{12}=0 \neq \beta_{1}=\beta_{2}$. Thus the $\beta_{\mathbf{j}}$ terms corresponding to areas common to more than one colorant, in general may vary with change in misregistration and may correspondingly produce a variation in the average spectrum for the halftone in (2).

If the terms $\beta_{\mathbf{j}}$ are invariant to inter-separation misregistration, we see from (2) that the average spectrum (and therefore the color) predicted by the model will also be invariant to inter-separation misregistration. Note that this is a sufficient but not necessary condition, i.e., invariance of the terms $\beta_{\mathbf{j}}$ 's to inter-separation misregistration ensures that the spectrum modeled in (2) is also invariant to misregistration. However, if the $\beta_{\mathbf{j}}$ 's show variation with registration, the average spectrum may still be invariant to misregistration errors- for example if the colorants display ideal behavior and have no unwanted absorptions. Given this nature of the interactions, we follow a two-fold approach to model misregistration sensitivity of a halftone configuration:

- First, in Sections 3.1 and 3.2 we examine the sensitivity of the terms $\beta_{\mathbf{j}}$ to registration. In general, the variation or lack thereof in the terms $\beta_{\mathbf{j}}$ with misregistration is determined by the halftone spot functions for the corresponding colorants (which also incorporates the area coverages for these colorants) as well as the periodicity lattices for the individual separations. However, for certain lattice configurations for the colorants, the terms are invariant to misregistration irrespective of the halftone spot function. We characterize these configurations in our asymptotic analysis.

- Next, in Section 3.3 we develop the complete model illustrated in Fig. 1 for the quantitative estimation of color shift due to misregistration. For this purpose, we utilize analytic forms of the spot function that allow simple (numerical) determination of the overlap areas $\beta_{\mathbf{j}}$ for any combination of separations.

\subsection{Lattice Analysis of Color Halftones}

The color halftone is obtained by superposing individual halftone screens whose individual periodicity is represented by the lattices $\left\{\boldsymbol{\Lambda}_{i}\right\}_{i=1}^{K}$. If the intersection of the resulting lattices $\boldsymbol{\Gamma}=\boldsymbol{\Lambda}_{1} \cap \ldots \cap \boldsymbol{\Lambda}_{K}$ is also a two dimensional lattice, then one can see that a period of $\boldsymbol{\Gamma}$ includes a period of each of the constituent lattices. Therefore the resulting superposition is also periodic with periodicity determined by the lattice $\boldsymbol{\Gamma}$. It can be shown that $\boldsymbol{\Gamma}$ is a two-dimensional lattice if and only if $\mathbf{V}_{j}^{-1} \mathbf{V}_{k}$ is a matrix of rational numbers for each $j, k \in\{1, \ldots K\}$. This condition on the lattices is termed commensurability by Amidror et al.. ${ }^{17}$

In practice for typical digital printing devices, the addressable device locations are confined to a rectilinear grid, and the $x$ and $y$ coordinates of the vectors $\mathbf{v}_{1}^{i}, \mathbf{v}_{2}^{i}$ are constrained to take values that are multiples of the corresponding grid spacing along these two directions. Consequently, it can be seen that the aforementioned condition is always satisfied. We therefore assume that this condition holds in our discussion and consider situations of interest where the condition may not be applicable as limiting cases.

We illustrate the above statements by means of an example in Fig. 2 for the case of $K=2$ halftones that are periodic on two orthogonal lattices $\boldsymbol{\Lambda}_{\mathbf{1}}$ and $\boldsymbol{\Lambda}_{\mathbf{2}}$ with basis matrices $\mathbf{V}_{1}=\left[\begin{array}{rr}2 & -4 \\ 4 & 2\end{array}\right]$ and $\mathbf{V}_{2}=$ $\left[\begin{array}{rr}4 & -2 \\ 2 & 4\end{array}\right]$, respectively. For this configuration, since $\mathbf{V}_{\mathbf{1}}^{-\mathbf{1}} \mathbf{V}_{\mathbf{2}}=\left[\begin{array}{rr}0.6 & 0.8 \\ -0.8 & 0.6\end{array}\right]$ is a matrix of rational numbers, the intersection of $\boldsymbol{\Lambda}_{\mathbf{1}}$ and $\boldsymbol{\Lambda}_{\mathbf{2}}$ is a two-dimensional lattice. The black circles in Fig. 2.c shows where the two lattices intersect. The union of the two lattices is the complete set of points indicated in Fig. 2.c where the colors of the points represent the lattices they originate from. From this the periodicity of the overlay is apparent. 


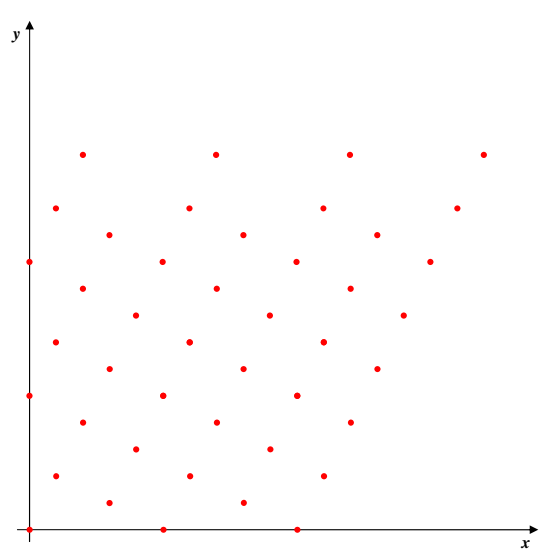

(a)

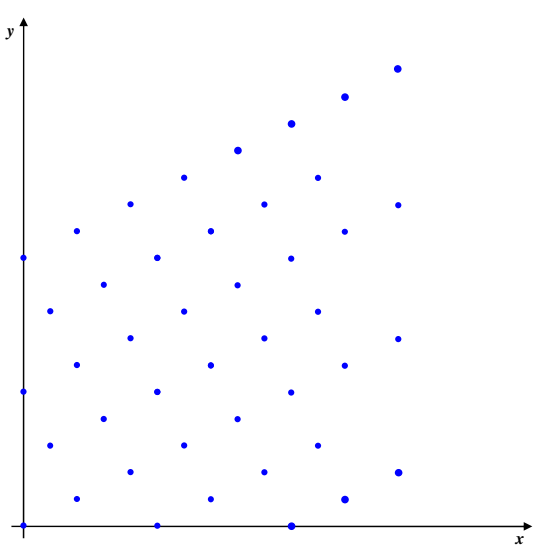

(b)

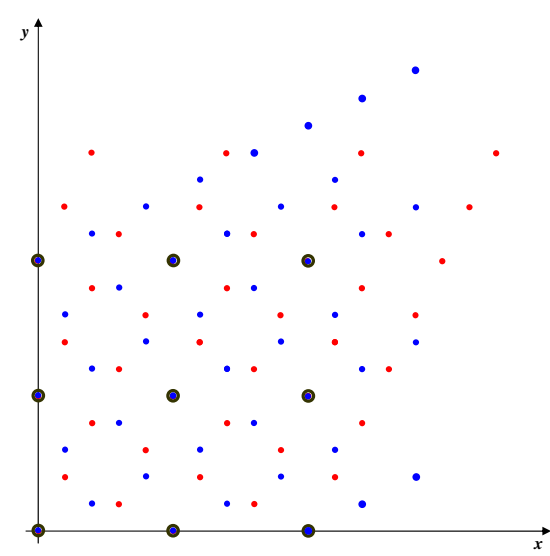

(c)

Figure 2. Example of lattice intersection. (a) \& (b) shows some portion of $\boldsymbol{\Lambda}_{\mathbf{1}}$ and $\boldsymbol{\Lambda}_{\mathbf{2}}$ respectively, the intersection of the lattices $\boldsymbol{\Gamma}=\boldsymbol{\Lambda}_{\mathbf{1}} \cap \boldsymbol{\Lambda}_{\mathbf{2}}$ shown with the black circles in in (c)

\subsection{Asymptotic Analysis of Misregistration Errors}

In Sec. 3 we pointed out the average color of the color halftone is a function of the fractional area coverage of each Neugebauer primary. Computing these terms requires calculating the overlap area of each possible halftone spot function overlay. As we pointed out in Sec. 3.1 under suitable constraints on the halftone periodicity lattices, the superposition of halftones is also periodic and computing the overlap areas of separations in a single period of the color halftone would be enough to characterize the overlap areas in the entire color halftone.

In order to simplify the discussion, we consider the superposition of two halftones that are periodic on lattices $\boldsymbol{\Lambda}_{\mathbf{1}}$ and $\boldsymbol{\Lambda}_{\mathbf{2}}$, respectively. As indicated earlier, the halftones may be represented by the convolution of the halftone dot function with the corresponding lattices. Without loss of generality, we assume that the halftone spot functions for the colorants are zero outside the confines of a unit cell and associate each replica of the halftone spot in a separation with a corresponding lattice point and therefore refer to halftone dot locations and lattice points interchangeably in the following discussion. Now, the halftone dot locations for the first halftone are the points $\mathbf{u}_{1} \in \boldsymbol{\Lambda}_{\mathbf{1}}$ and the halftone dot locations for the second halftone are the points $\mathbf{u}_{2} \in \boldsymbol{\Lambda}_{\mathbf{2}}$. The set of all possible displacements between a dot location in the first halftone and a dot location in the second halftone is therefore the set

$$
\left\{\mathbf{u}_{1}-\mathbf{u}_{2} \mid \mathbf{u}_{1} \in \boldsymbol{\Lambda}_{1}, \mathbf{u}_{2} \in \boldsymbol{\Lambda}_{2}\right\}=\left\{\mathbf{u}_{1}+\mathbf{u}_{2} \mid \mathbf{u}_{1} \in \boldsymbol{\Lambda}_{1}, \mathbf{u}_{2} \in \boldsymbol{\Lambda}_{2}\right\}=\boldsymbol{\Lambda}_{1}+\boldsymbol{\Lambda}_{2}
$$

where the second step follows from the fact that for every $\mathbf{u}_{2} \in \boldsymbol{\Lambda}_{2}$ we have $-\mathbf{u}_{2} \in \boldsymbol{\Lambda}_{2}$. Under our assumption that $\boldsymbol{\Gamma}$ is a two-dimensional lattice, the set $\boldsymbol{\Lambda}_{1}+\boldsymbol{\Lambda}_{2}$ is also a two-dimensional lattice. Since only these discrete displacements are observed between the two lattices, we can determine the area $\beta_{12}$ which is common to the two halftones in terms of these displacements and the intersection area for dot shapes at these displacements. Denoting by $\alpha(\boldsymbol{\nu})$ the area common between a halftone spot of the first halftone and a halftone spot of the second halftone with a displacement $\boldsymbol{\nu}$ between them. Now consider a basis $\mathbf{v}_{1}, \mathbf{v}_{2}$ for $\boldsymbol{\Gamma}$ and a corresponding unit cell defined as

$$
\Xi=\left\{\alpha \mathbf{v}_{1}+\beta \mathbf{v}_{2} \mid-0.5 \leq \alpha \leq 0.5,-0.5 \leq \beta \leq 0.5\right\}
$$

Then the fraction of area common to the two screens is

$$
\beta_{12}=\sum_{\boldsymbol{\nu} \in\left(\boldsymbol{\Lambda}_{1}+\boldsymbol{\Lambda}_{2}\right) \cap \Xi} \alpha(\boldsymbol{\nu})=\int_{\boldsymbol{\nu} \in \Xi} \alpha(\boldsymbol{\nu}) p(\boldsymbol{\nu}) d \boldsymbol{\nu}
$$

where $p(\boldsymbol{\nu})=\sum_{\boldsymbol{\nu} \in\left(\boldsymbol{\Lambda}_{1}+\boldsymbol{\Lambda}_{2}\right)} \delta(\boldsymbol{\nu})$, with $\delta()$ denoting the Heaviside delta function. 
Let $\beta_{12}^{\mathbf{d}}$ represent the overlap area of the screen displaced by a displacement vector $\mathbf{d}$. In this case, (8) can be modified such that $p(\boldsymbol{\nu})$ is shifted by an amount $\mathbf{d}$ as:

$$
\beta_{12}^{\mathbf{d}}=\int_{\boldsymbol{\nu}} \alpha(\boldsymbol{\nu}) p(\boldsymbol{\nu}-\mathbf{d}) d \boldsymbol{\nu} .
$$

From the above equation, we see that if $\int_{\boldsymbol{\nu}} \alpha(\boldsymbol{\nu}) p(\boldsymbol{\nu}-\mathbf{d}) d \boldsymbol{\nu}$ is independent of $\mathbf{d}$ the overlap area for the two halftones is independent of the misregistration. Under the restrictions adopted in the analysis above, we see this does not hold for arbitrary $\alpha(\boldsymbol{\nu})$ because $p(\nu)$ is in fact a finite discrete distribution. For illustrative purposes, we calculate and depict the term $p(\nu)$ for the superposition of two one-dimensional lattices. Fig. 3 illustrates an example for the intersection of two one-dimensional lattices at different displacements. The distributions $p(\boldsymbol{\nu})$ and $p(\boldsymbol{\nu}-\mathbf{d})$ are clearly not equal to each other and this difference makes the superposition sensitive to misregistration.
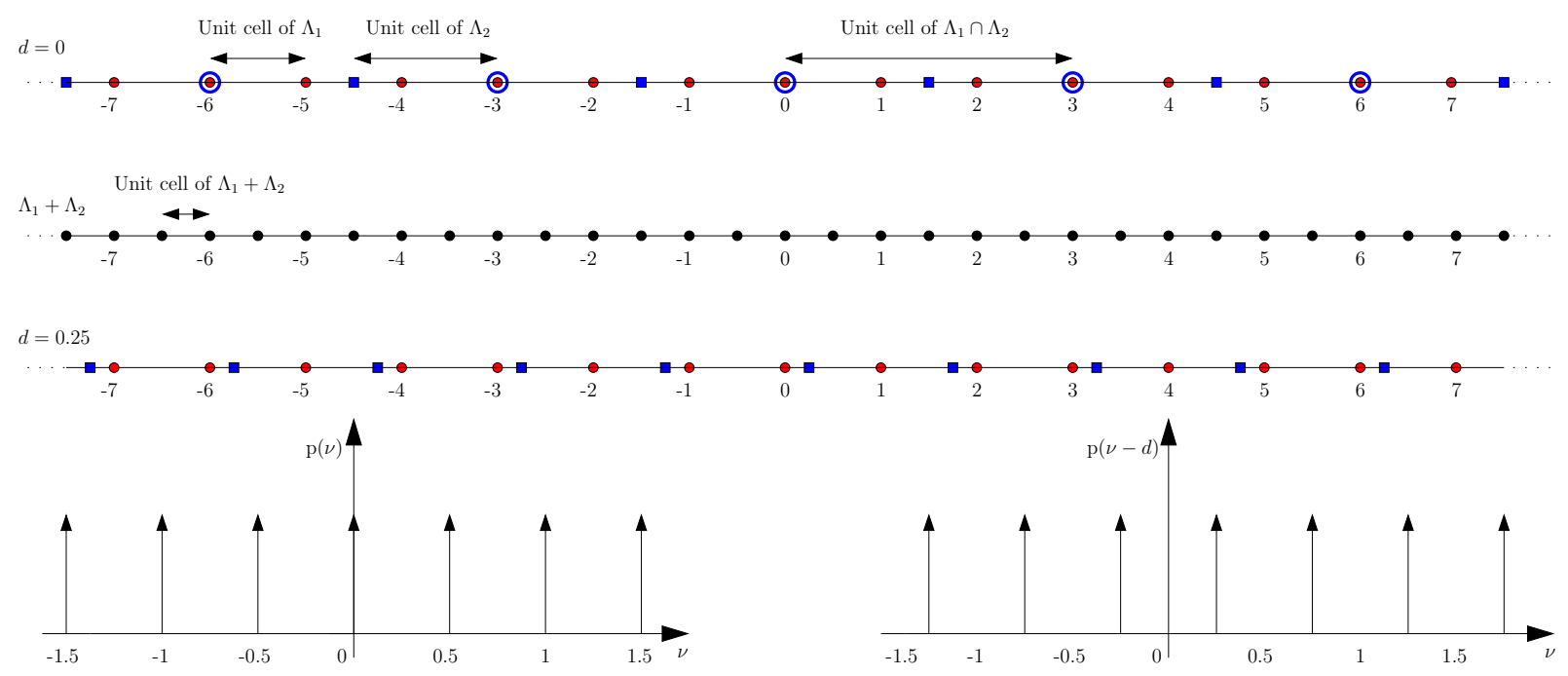

Figure 3. Example of superposition of two one-dimensional lattices at different displacements

Note that if $\alpha(\boldsymbol{\nu})$ is a continuous function (which will commonly be the case) and $p(\nu)=\sum_{\boldsymbol{\nu} \in\left(\boldsymbol{\Lambda}_{1}+\boldsymbol{\Lambda}_{2}\right)} \delta(\boldsymbol{\nu})$ is dense in the (two-dimensional) set $\Xi$, then we see that $\int_{\boldsymbol{\nu}} \alpha(\boldsymbol{\nu}) p(\boldsymbol{\nu}-\mathbf{d}) d \boldsymbol{\nu}$ is independent of $\mathbf{d}$ and the superposition of the two halftones becomes invariant to registration. From an engineering perspective, allowing for some jitter in position of the individual halftones this mathematical condition may be approximated by a sufficient 2-D denseness requirement.

Note that mathematically, the halftones exhibit sensitivity to registration provided there exist non-zero $\mathbf{n}_{1} \in \mathbb{Z}^{2}$ and $\mathbf{n}_{2} \in \mathbb{Z}^{2}$ such that $\mathbf{V}_{1} \mathbf{n}_{1}+\mathbf{V}_{2} \mathbf{n}_{2}=0$, a condition referred to as singular state. ${ }^{10}$ If no such integer values exist then $\Gamma$ is aperiodic (along all directions), the distribution $p(\nu)$ is dense and the halftone overlaps are invariant to registration.

\subsection{Color Halftone Model}

Clustered dot halftones are generated by comparing the image values $\tau(x, y)$ against a periodic halftone threshold array $K_{T}(x, y)$. This threshold array was conventionally defined as a discrete array of thresholds but alternatively it can be represented by an analytic threshold function. Pellar ${ }^{18,19}$ defined such a threshold function as:

$$
K_{T}(x, y)=\frac{\cos \left(2 \pi f_{x} x\right)+\cos \left(2 \pi f_{y} y\right)+2}{4},
$$

where $f_{x}$ and $f_{y}$ are the screen frequencies, $x$ and $y$ are the spatial positions. 
This function can be modified such that it incorporates screen orientation and displacement as:

$$
K_{T}(x, y ; \phi, \Delta x, \Delta y)=\frac{\cos \left(2 \pi f x^{\prime}\right)+\cos \left(2 \pi f y^{\prime}\right)+2}{4},
$$

where

$$
\left[\begin{array}{l}
x^{\prime} \\
y^{\prime}
\end{array}\right]=\left[\begin{array}{rr}
\cos (\phi) & -\sin (\phi) \\
\sin (\phi) & \cos (\phi)
\end{array}\right] \times\left[\begin{array}{l}
x-\Delta x \\
y-\Delta y
\end{array}\right]
$$

$\phi=\tan ^{-1}\left(\frac{v_{y}}{v_{x}}\right)$ is the rotation angle of the screen and $\Delta x$ and $\Delta y$ are the registration errors along horizontal and vertical directions respectively, and the other terms are defined before.

Computing the fractional dot area coverage involves integrating the function in (11). Although numerical approximations to the integral can be found, it is hard to represent it in an exact analytic form. Moreover, computation of inter-separation overlap areas is further complicated by finding the intersection points of multiple functions of this type. To simplify this, we observe the dot growth sequence the halftone obtained by thresholding (11) in Fig. 4. While the transition from highlights to midtones can be characterized as growing black dots, after the fractional area coverage reaches 0.5 the transition from midtones to shadows can be represented as shrinking white holes. Furthermore, these dots and holes can be generated by thresholding an analytical function of the form

$$
|x|^{k}+|y|^{k}=r^{k}
$$

where $k \in \mathbb{R}$ and $k \geq 1$. Although the integral of this function can be found using integral tables, in this paper we further simplify this computation by choosing two special forms of (13). At highlights and shadows we choose $k=2$ yielding circular dots and holes, and at midtones we use $k=1$ generating square dots and holes.

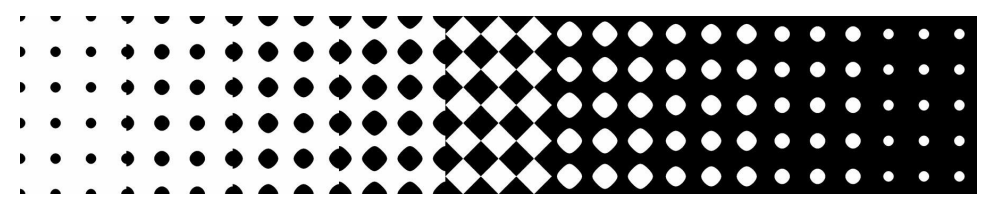

Figure 4. Dot growth with threshold function defined by (11)

A visual closeness metric is employed to determine the optimal value for the fractional area coverage of the screen bounding the midtone gray levels denoted by $\mu$. Since the dots and holes are of same size for the fractional area coverages $\beta$ and $1-\beta$ respectively, we carry on to find this optimum value in the range $\beta \in[0,0.5]$. For a given $\beta$, let $\mathbf{D}_{\text {cos }}^{\beta}$ denote the dot obtained by thresholding (11). The size of the square or circle that approximates $\mathbf{D}_{\text {cos }}^{\beta}$ visually varies between the size of the square or circle that is circumscribed by $\mathbf{D}_{\text {cos }}^{\beta}$ and that circumscribes $\mathbf{D}_{\text {cos }}^{\beta}$. Using computer simulations on a high resolution grid, a large sample of square and circular dots having sizes within the specified range are generated and overlapped with $\mathbf{D}_{\text {cos }}^{\beta}$. An error metric namely fractional area coverage error is formulated as the non-overlapping area between $\mathbf{D}_{\cos }^{\beta}$ and the other dots. The dot having the least error is chosen to represent $\mathbf{D}_{\text {cos }}^{\beta}$. Fig. 5 shows the fractional area coverage error versus $\beta$ for both square and circular dots.

As expected from Fig. 4, at highlights and shadows circular dots have less error than square dots, and at midtones square dots have less error than circular dots. At approximately $\mu=0.413$ the error curves for the square and circular dots intersect. This value corresponds to the optimal value of $\beta$ determining the dot shape representing $\mathbf{D}_{\text {cos }}^{\beta}$.

\subsection{Dot Overlap Model of Color Halftones}

As it is known from (2), the fractional area coverage of each Neugebauer primary is needed to exercise the Neugebauer equations to find the average spectrum of the color halftone.. In Sec. 3.2 we discuss the total fractional overlap area can be obtained by averaging the overlap areas of each possible inter-separation dot intersection weighted by the displacement distribution function obtained by lattice analysis of color halftones. 


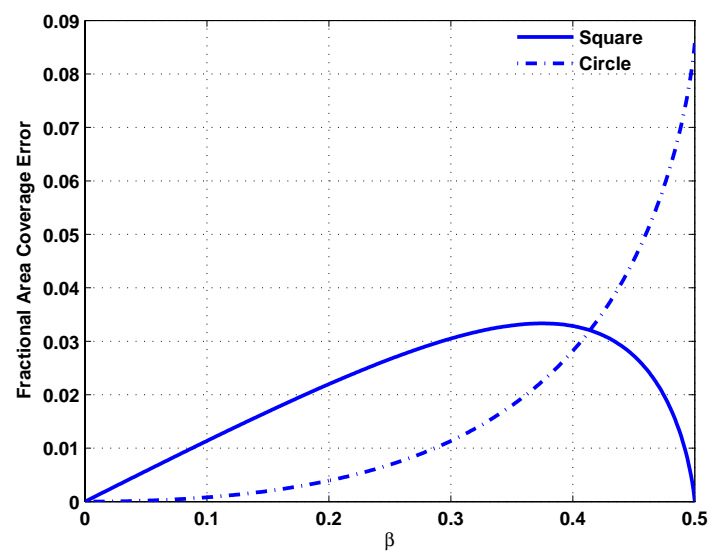

Figure 5. Fractional area coverage error between best square and circle approximation to $\mathbf{D}_{\cos }^{\beta}$ and $\mathbf{D}_{\cos }^{\beta}$

In Sec. 3.3, circle and square dots are shown to be appropriate to approximate the dot shapes obtained from a known analytic threshold function. In the current study, we assume each colorant has the same fractional area coverage. Thus, only the intersections of circles or squares are assumed in this analysis. Rogers ${ }^{9}$ showed how to find the intersection area of two circular dots analytically as a function of the distance between their centers and their radii. That work can be extended to the intersection of $K$ circular dots by keeping track of the coordinates of the intersection points of pairs of circles. For the intersection of square dots, Sutherland-Hodgman polygon clipping method ${ }^{20}$ is useful to find the vertices of the intersection polygon. An example of finding the intersection polygon of two squares is shown in Fig. 6. To find the intersection polygon, one of the squares is clipped with the edges of the other square sequentially. Convex polygon triangulation method is then used to find the intersection area of the polygon. The total area is simply the sum of the individual areas of the triangles that form the polygon and the areas of the triangles can easily be computed since the coordinates of the vertices are known.

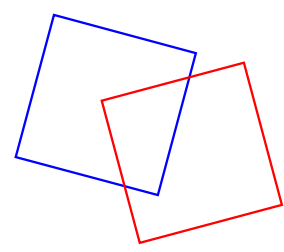

(a)

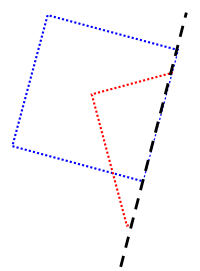

(b)

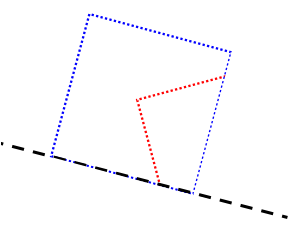

(c)

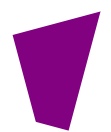

(d)

Figure 6. Example of Sutherland - Hodgman polygon clipping. (a) shows two squares before clipping, (b) and (c) shows the intermediate steps, (d) shows the intersection polygon

\subsection{Calculation of the Average Color Shift}

After the fractional areas, $a_{c_{1} \ldots c_{K}}$ 's, are determined by the method described in Sec. 3.3, we find the average spectrum of the color halftone by using (2). Next, we calculate the XYZ value using the average spectrum and then Lab value of to color halftone is determined by using the XYZ value. ${ }^{2}$

The same computation is repeated for the estimation of the average color of the reference color halftone and another Lab value is obtained. Finally, we calculate the average color change in $\Delta E_{a b}^{*}$ units by computing the Euclidean distance between the Lab pairs. 


\section{EXPERIMENTAL RESULTS}

We carried out several experiments to validate our misregistration analysis model. Several test targets were generated with different amounts of misregistration deliberately introduced in the halftone separations and measurements of these were compared against predictions from the model. Since inter-separation misregistration is unavoidable even in experimental prints and often locally varying, the precise determine the amount of actual inter-separation misregistration in the prints is unknown. Therefore we estimate the maximum, mean and the minimum average color shift among all average color shifts between the target pairs in $\Delta E_{a b}^{*}$ units and compare these values with our model's maximum average color shift estimate for that orientation.

Since midtones were found to be the most sensitive among two colorant configurations ${ }^{13}$ and the halftones are well approximated by square dots in these regions (see Section 3.4) we utilize a square dot shape for both our experimental prints as well as our model that is obtained by a separable combination of two saw-tooth threshold functions. The test targets are generated using halftone lattices on the $4800 \times 600$ dpi printer addressability grid.

For our prints and simulations we utilize the a halftone configuration with orthogonal CMYK screens whose lattice bases (upto scale factor) are listed in Table 1. These are oriented as $\mathbf{C}$ at $\tan ^{-1}(3) \simeq 71.565^{\circ}, \mathbf{M}$ at $\tan ^{-1}\left(\frac{1}{3}\right) \simeq 18.435^{\circ}, \mathbf{Y}$ at $\tan ^{-1}(0)=0^{\circ}$, and $\mathrm{K}$ at $\tan ^{-1}(1)=45^{\circ}$. These angles are chosen to approximate the conventional $30^{\circ}$ difference between $\mathbf{C}, \mathbf{M}$ and $\mathbf{K}$ screens. Using this set of screens, we test the misregistration sensitivity of superposition of all two colorant pairs and also the average color shift between the 3-color CMK dot-centered and hole-centered rosettes. For each of the two colorant cases, the print included samples in which the inter-separation misregistration that was electronically introduced ranged over a cell. For the 3color configuration, since the number of possible inter-separation misregistrations is rather large only these two configurations are utilized and no additional misregistration is introduced in the printed targets. For each colorant, we aimed for a fractional area coverage of 0.5 in the test target (and used the same for our simulations). This is accomplished by estimating the area coverages for the colorants and pre-compensating for variation in the printer physical response. ${ }^{13}$ Finally, each test target is printed multiple times and measured using a spectrophotometer.

\begin{tabular}{|c|c|c|c|}
\hline $\mathbf{V}_{C}$ & $\mathbf{V}_{M}$ & $\mathbf{V}_{Y}$ & $\mathbf{V}_{K}$ \\
\hline$\left[\begin{array}{rr}2 & -6 \\
6 & 2\end{array}\right]$ & {$\left[\begin{array}{rr}6 & -2 \\
2 & 6\end{array}\right]$} & {$\left[\begin{array}{ll}4 & 0 \\
0 & 4\end{array}\right]$} & {$\left[\begin{array}{rr}4 & -4 \\
4 & 4\end{array}\right]$} \\
\hline
\end{tabular}

Table 1. Lattice Bases for the CMYK color halftone configuration.

For our simulations, we also print the $2^{4}=16$ Neugebauer primaries using the same printer we printed the test targets and measured their spectrum. The Yule-Nielesen parameter was estimated as $\gamma=2.5^{13}$ and utilized throughout the simulations. The fractional area coverages of the screens are taken equal to each other and we run our simulations for $\beta$ equal to 0.4, 0.5 and 0.6. For each orientation, we determine the maximum attainable $\Delta E_{a b}^{*}$ running the simulations over a large sample of displacements. However, only for the dot-centered rosettes versus hole-centered rosettes comparison, we did not consider any registration errors on the screens and compute the $\Delta E_{a b}^{*}$ for the given $\beta$ 's. The results are shown in Table 2 .

From the table one can see that the model estimates are in qualitative agreement with the experimental results. However, quantitatively the simulation results do not match the experimental measurements. There are several reasons for these discrepancies. Firstly, observe that at $\beta=0.5$ the model estimate of the color shift for all colorant combinations except for the CM colorant pair is zero. This arises due to the specific halftone periodicities combined with the halftone dot shape that ensure invariance of the overlap areas despite the discrete distribution of dot displacements. In practice however, the dot shapes will deviate from the exact squares used in our simulation and additionally will be affected by the pre-compensation process. For this reason, the color shifts for values of $\beta=0.4$ and 0.6 are also included in Table 2. Secondly, the unknown misregistration in the experiments also leads to uncertainty regarding the relation between the measurements and simulations. 


\begin{tabular}{|c|c|c|c|c|c|c|}
\hline \multirow{2}{*}{ Colorants } & \multicolumn{3}{|c|}{ Measured $\Delta E_{a b}^{*}$} & \multicolumn{3}{c|}{ Model Estimate Max. } \\
\cline { 2 - 4 } & Max & Mean & Min & \multicolumn{3}{|c|}{$\Delta E_{a b}^{*}$} \\
\hline CM & 2.9676 & 0.8858 & 0.0334 & 1.2512 & 1.5676 & 1.2713 \\
CY & 2.5314 & 0.9331 & 0.0711 & 0.2329 & 0 & 0.2310 \\
CK & 3.9262 & 1.5065 & 0.0257 & 0.2010 & 0 & 0.2057 \\
MY & 3.7051 & 1.1597 & 0.0373 & 0.8659 & 0 & 0.9167 \\
MK & 4.2289 & 0.9829 & 0.0289 & 0.2481 & 0 & 0.2574 \\
YK & 7.0910 & 2.5172 & 0.0652 & 7.5346 & 0 & 7.8895 \\
CMK & 3.4559 & 1.1349 & 0.0339 & 0.2307 & 0 & 0.2389 \\
\hline
\end{tabular}

Table 2. Measured $\Delta E_{a b}^{*}$ values and model estimate for the given colorant pairs

As it can be seen that the model predicts misregistration insensititivity for fractional area coverages $\beta=0.5$ but fractional area coverages of 0.4 and 0.5 show significant registration sensitivity. The magnitude of predicted shifts in color (in $\Delta E_{a b}^{*}$ ) are consistent with the corresponding ranges obtained in experiments.

The results illustrate the complicated nature of the inter-separation misregistration problem. For any given configuration, the sensitivity is dependent on: a) spectral colorant interactions and b) spatial halftone separation interactions. The latter in turn depend in a complicated fashion on both the halftone periodicities as well as the specific geometrical size and shape of the dots. The model developed here provides a method for probing these dependencies. The preliminary results presented here are qualititively consistent with the experimental meaurements. In our continuing work we are exploring further validation of the model both in qualitative and quantitative aspects.

\section{CONCLUSIONS}

In this paper, we examined the effect of inter-separation misregistration on the average color obtained from the superposition of periodic halftone screens. We indicate that a complete understanding of the impact of misregistration requires analysis of both spatial and spectral interactions between the separations. We developed a framework for quantitative estimation of misregistration induced color shifts using a lattice representation. The lattice representation allows an intuitive development of existing results on misregistration sensitivity for the spatial interactions and also provides a computationally efficient model for the quantitative estimation of color shifts. We demonstrate that the model provides qualitative agreement with experimental measurements.

\section{REFERENCES}

1. J. A. C. Yule, Principles of color reproduction, applied to photomechanical reproduction, color photography, and the ink, paper, and other related Industries, Wiley, New York, 1967.

2. G. Sharma, "Color fundamentals for digital imaging," in Digital Color Imaging Handbook, G. Sharma, ed., CRC Press, Boca Raton, FL, 2003. Chapter 1.

3. F. A. Baqai, J.-H. Lee, A. U. Agar, and J. P. Allebach, "Digital color halftoning," IEEE Sig. Proc. Mag. 22, pp. 87-96, Jan. 2005.

4. W. H. F. Talbot, "Improvements in the art of engraving." British Patent Specification No. 565, 29 Oct. 1852.

5. H. E. J. Neugebauer, "Die theoretischen Grundlagen des Mehrfarbenbuchdrucks," Zeitschrift für wissenschaftliche Photographie Photophysik und Photochemie 36, pp. 73-89, Apr. 1937. reprinted in. ${ }^{21}$ 
6. J. A. S. Viggiano, "Modeling the color of multi-colored halftones," TAGA Proc., pp. 44-62, 1990.

7. E. Demichel in Procédé, 26, pp. 17-21,26-27, 1924.

8. R. Balasubramanian, "A printer model for dot-on-dot halftone screens," in Proc. SPIE: Color hard copy and graphic arts IV, J. Bares, ed., 2413, pp. 356-364, Feb. 1995.

9. G. L. Rogers, "Neugebauer revisited: Random dots in halftone screening," Color Res. Appl. 23, pp. 104-113, Jan. 1998.

10. I. Amidror and R. D. Hersch, "Neugebauer and demichel: Dependence and independence in n-screen superpositions for colour printing," Color Res. Appl. 25, pp. 267-277, Aug. 2000.

11. I. Amidror and R. D. Hersch, "Analysis of the microstructures ("rosettes") in the superposition of periodic layers," J. Electronic Imaging 11, pp. 316-337, Jul. 2002.

12. K. Daels and P. Delabastita, "Color balance in conventional halftoning," in TAGA Proc., pp. 1-18, 1994.

13. B. Oztan, G. Sharma, and R. P. Loce, "Quantitative evaluation of misregistration induced color shifts in color halftones," in Proc. SPIE: Color Imaging X: Processing, Hardcopy, and Applications, R. Eschbach and G. G. Marcu, eds., 5667, pp. 501-512, Jan. 2005.

14. J. W. S. Cassels, An Introduction to the Geometry of Numbers, Springer, first ed., 1959.

15. T. S. Rao and G. R. Arce, "Halftone patterns for arbitrary screen periodicities," J. Opt. Soc. Am. A 5, pp. 1502-1511, Sept. 1988.

16. T. S. Rao, G. R. Arce, and J. P. Allebach, "Analysis of ordered dither for arbitrary sampling lattices and screen periodicities," IEEE Trans. Acoust. Speech Sign. Proc. 38, pp. 1981-2000, Nov. 1990.

17. I. Amidror and R. D. Hersch, "Analysis of the superposition of periodic layers and their moiré effects through the algebraic structure of their fourier spectrum," J. Math. Img. and Vision 8, pp. 99-130, 1998.

18. R. J. Pellar and L. Green, "Electronic halftone generator." United States Patent No. 4149 183, 1979.

19. R. J. Pellar, "Electronic halftone generator." United States Patent No. 4196 451, 1980.

20. I. E. Sutherland and G. W. Hodgman, "Reentrant polygon clipping," Commun. ACM 17, pp. 32-42, Jan. 1974.

21. K. Sayangi, ed., Proc. SPIE: Neugebauer Memorial Seminar on Color Reproduction, vol. 1184, SPIE, Bellingham, WA, 14-15 Dec. 1989. 